\section{Immersive Design: drawing and perspective through technological devices of Virtual Reality}

\section{Rodrigo Ayabe}

Universidade Anhembi Morumbi

The human experience is a multisensory process, which shifts according to changes in perception and individual experiences. Experiencing an architectural environment involves these nuances derived from living with a place. This concept of immersion in the environment to promote multisensory stimuli receives from Jon Charles Coe (2012) the name of "Immersive Design", a term derived from architecture, but applied also to Virtual Reality(VR), Augmented Reality, Mixed Reality, Extended Reality, as well as structuring languages for object-oriented design and Human-Computer-Interaction.

Since the Renaissance technology has become an essential element in the perception of the gaze thanks to the development of optical devices and techniques for representing the perspective. The result can be seen in the paintings, with detailed depictions of depth and textures, as well as in the architecture of cathedrals and other buildings, enabling higher and wider indoor spaces.

Contemporaneously, based on our own experiences that of other professors, we have identified major problems in teaching perspective and spatial reasoning in drawing classes, either due to the previous deficiency in geometry, or due to the majority use of two-dimensional technologies - such as screens, displays and paper. This scenario can restrict the drawing classes experience, the realization of projects, and even discourage the teaching-learning process.

The research "Immersive Design, through technological devices of Virtual Reality", has worked on different methodologies mediated by the use of VR devices for teaching perspective in drawing classes. Among "digital natives" students the use of VR has stimulated new forms of perception and sensitivity thanks to the three- dimensional experience in immersive environments.

In the virtual space the student can experience in an empirical way the perspective involved in the construction of spatial elements. This happens, for example, with the possibility of drawing directly in three-dimensional space and "walking" along the trace - even controlling the time of the action. Therefore, it becomes possible to unveil the image from various angles by displacing the observer, unlike "static" techniques structurally limited to flat and two-dimensional supports.

Mediated by VR apparatuses the body immerses itself in the digital, creating a synergy between the gestural and the conceptual in three-dimensional virtual environments, that can function simultaneously as a space for representation and embodiment. According to Philippe Quéau (1996), the image becomes an exploitable place that extrapolates its representational condition. The image is no longer a substrate within the experience would be written. This place is itself an image - a kind of symptom of the symbolic model that is at its origin. It is the very experience of that space that allows us to return to the source of its intelligibility, that is, to the model. This is the experience that epigenetically constitutes it as "space". The synthetic image modifies our relationship with the immediate reality, structuring it as a "drawable" instrument.

The analysis of the results validates the research hypotheses and has allowed to rescue didactic and perceptual principles related to the perspective. In addition, it indicates new perspectives and potentialities for the use of VR in education, especially, but not limited to, drawing classes.

KEYWORDS

Virtual Reality, Immersive Design, Drawing, Perspective, Drawing Classes 